\title{
Catatonia in a woman who is profoundly deaf-mute: case report
}

\author{
Gbolagade Sunmaila Akintomide, ${ }^{1}$ Stuart Williams Porter, ${ }^{1}$ Anita Pierce ${ }^{1}$
}

The Psychiatrist (2012), 36, 418-421, doi: 10.1192/pb.bp.112.038638

${ }^{1}$ Betsi Cadwaladr University Health Board, North Wales

Correspondence to Gbolagade Sunmaila Akintomide

(gbolagade.akintomide@nhs.net)

First received 17 Jan 2012, final revision 20 Mar 2012, accepted 23 Apr 2012
Summary Catatonia is a common but underrecognised complication of bipolar disorder, with a quarter of in-patients with bipolar disorder developing catatonia. Almost 9 million people in the UK are deaf or have a significant hearing problem and British Sign Language is the preferred language of 50000-70 000 people within the UK. Between 1 and $2 \%$ of these individuals (i.e. the same as in the rest of the population) will experience bipolar disorder in their lifetime and therefore the accurate diagnosis of catatonia is important. We report a case of catatonia presenting with dysphagia in a woman who is profoundly deaf-mute and who has bipolar disorder. This report highlights some modifications of presentation and difficulties of accurate diagnosis and management of catatonia in this patient. To the best of our knowledge, this is the first case report of catatonia in someone who is profoundly deaf-mute.

Declaration of interest None.

\section{Case report}

We report, with the patient's consent, on the case of a 48-year-old woman who is profoundly deaf-mute and who communicates exclusively with British Sign Language. She has a 7-year history of bipolar affective disorder. She had been covertly non-adherent to taking her psychotropic medication, for 6 months prior to her presentation. During this time she experienced significant psychosocial stressors. A month before presentation, she was prevented from impulsively jumping in front of a moving train when a relative failed to collect her from a train station.

During a domiciliary visit, she was found to have low mood, poor appetite and self-care. With the aid of the interpreter she suggested she was afraid to be in the house alone. She reported that there were people entering her house who were not her family. She could provide no evidence to support this belief. She was preoccupied that a tree had started to grow outside her window that had significance for her and was frightening. She struggled to describe the idea in greater detail. In response to these ideas she insisted on keeping the curtains closed at all times. She was thought to have a depressive episode with psychotic symptoms and was prescribed olanzapine $5 \mathrm{mg}$ and duloxetine $60 \mathrm{mg}$.

During the following week she remained non-adherent to taking her medication. She developed constipation and difficulty in swallowing. Her mental state demonstrated increasing paranoid ideation, believing her drinking water had been poisoned. She was subsequently admitted informally to an acute psychiatric unit. On admission, she was found to be tachycardic, severely dehydrated and regurgitating fluid on attempted swallowing. She appeared to have developed nihilistic delusion, expressing the idea that if she drank, the water would never leave her body and this would harm her. She was transferred to the acute medical unit of a nearby general hospital.
On admission to the general hospital, she was found to have acute renal failure secondary to dehydration. She was also found to be constipated with faecal impaction. She was rehydrated with her renal function normalising. She was found to have raised inflammatory markers. By the third day in the general hospital she had stopped communicating with sign language even with the aid of an interpreter. Speech and language therapist evaluation revealed difficulty at the pharyngeal phase of swallowing; they recommended she be nursed nil by mouth. Following an ear, nose and throat opinion, a diagnosis of acute tonsillitis secondary to dehydration was made. She was started on antibiotics.

On her seventh day in hospital she continued to lack a swallow reflex. Her inflammatory markers including C-reactive protein and neutrophil were falling. She developed electrolyte derangement with low potassium $(2.9 \mathrm{mmol} / \mathrm{l})$. She was started on nasogastric tube feeding, her intravenous correction of the electrolyte imbalance continued, with the addition of thiamine via nasogastric tube and intravenous multivitamins (pabrinex). Her psychiatrist suspected depression with psychotic symptoms and she was prescribed olanzapine $10 \mathrm{mg}$ and mirtazapine $30 \mathrm{mg}$ via nasogastric tube.

Within 24 hours of starting nasogastric tube feeding, she developed low phosphate and magnesium of 0.58 and $0.49 \mathrm{mmol} / \mathrm{l}$ respectively, with sinus tachycardia of $102 / \mathrm{min}$. She was suspected to have developed refeeding syndrome and the nasogastric tube feeding was promptly suspended. On the ninth day it was noted during a review by the medical team that she had posturing of her right hand, increased muscle tone and cog wheel rigidity in both upper limbs. She was found to have an inverted right foot and persistently pursed mouth. It was suspected to be either a 
dystonic reaction to olanzapine or a complication of electrolyte imbalance. The latter was later excluded.

On the same day, she was reviewed by the psychiatric team who interpreted the clinical picture differently. We noted persistent lack of communication, aspontaneity, trismus (pursed mouth) and stupor (voluntary motionlessness). She was not felt to have rigidity but rather negativism (resistance to passive movement of her limbs.) We felt her presentation was in keeping with catatonia with a differential diagnosis of neuroleptic malignant syndrome. Olanzapine was stopped, her serum creatinine phosphokinase was found to be normal. She was started on a trial of diazepam via a nasogastric tube as lorazepam is not available in liquid preparation. She was reviewed by a neurologist who also found waxy flexibility and depressed reflexes without accompanying generalised weakness. Her computed tomography (CT) scan was normal.

Owing to her repeatedly pulling out her nasogastric tube, resulting in inconsistent administration of her medication and nutrition, she was assessed and found not to have the capacity to give consent to insertion of a bridle nasogastric tube. This was inserted in her best interest on the sixteenth day in the general hospital. She was treated with high-dose diazepam.

By the twenty-third day, after six days of consistent diazepam administration, she was still catatonic with dysphagia. A bedside nasoendoscopy demonstrated no mechanical cause or obstruction to explain her persisting lack of a swallowing reflex. By the twenty-seventh day, with no other explanation for the persistent dysphagia apart from catatonia, she was put on Sections 3 and 62 of the Mental Health Act and was prescribed emergency electroconvulsive therapy (ECT). An immediate improvement in her mood, communication, expressivity and spontaneity was noticed following the first session of ECT. The ECT was stopped after the third session; her mood was euthymic and apart from ongoing swallowing problems her catatonia had resolved. Improvement in her swallowing was relatively slow, although she had started making efforts to swallow. Eventually, she was able to swallow liquid and then solid food on the fifty-sixth and sixtieth day of her admission. Her diazepam was gradually stopped and sodium valproate was introduced. An electromyography done to rule out motor neuron disease revealed no abnormality. She was discharged home on the sixty-third day.

\section{Discussion}

We have reported a case of catatonia in a woman who is profoundly deaf-mute. The diagnosis was delayed on account of difficulties in communication with the patient, unusual early presentation with dysphagia and modification of common signs of catatonia. To the best of our knowledge there has been no known report of catatonia in a neurologically intact deaf person. The only case we found in the literature was syndromic perceptive deafness in association with diabetes, cerebral atrophy and mental weakness. ${ }^{1}$ Our case satisfied the DSM-IV-TR criteria for catatonia in mood disorders by having at least two features out of: waxy flexibility or stupor; negativism; posturing or grimacing; excessive motor activity and echolalia or echopraxia. ${ }^{2}$
The delay in diagnosis of at least 10 days was due to uncommon but known presentation with dysphagia ${ }^{3,4}$ and extra difficulties in detecting her catatonia features. The dysphagia was wrongly attributed to reluctance to drink secondary to persecutory and nihilistic delusion, and then to tonsillitis. The misinterpretation was the result of difficulties trying to get a detailed history from someone whose first language is British Sign language via an interpreter. However, the early presentation of the dysphagia and its delayed response to ECT (a delay of about 20 days, unlike the other features of her catatonia) raises the possibility that the dysphagia might not be part of her catatonia but instead it could be secondary to another unidentified physical or psychological cause. Nevertheless, the slow response of dysphagia to ECT has been reported elsewhere. ${ }^{5}$ Thus, the dysphagia most probably represents an interesting, albeit impossible to confirm, part of the catatonic spectrum in this patient. ${ }^{3,4}$

The other extra difficulties in diagnosis encountered because the patient was deaf-mute included (a) delays in recognising that reduced gesticulation of sign language signified 'muteness' of catatonia rather than deliberate uncooperativeness with the medical team (she has a history of being suspicious of medical personnel) and (b) initial wrong attribution of 'negativism' (resistance to passive limb movement) to rigidity, notwithstanding lack of concurrent weakness or hyperreflexia. There was a rather retrospective recognition of catalepsy after a diagnosis of catatonia had been made (in her own case, maintenance of raised hands in elevated positions for unusually long periods of time). It was rather the posturing of her right hand and foot that gave the diagnosis away.

The difficulties encountered in her management as a result of her being deaf-mute included the ethical and moral difficulties of taking an informed consent for insertion of a bridle nasogastric tube. Her repeatedly pulling out her nasogastic tube was rather suggestive of her not wanting one. Thus, we had to assess for capacity to consent to insertion of a bridle nasogastric tube with the help of an interpreter and she was found to lack capacity on account of 'impaired judgement'. The bridle nasogastric tube was inserted in her best interest after a discussion with her relatives.

She was belatedly given ECT 19 days after the diagnosis of catatonia was made because we felt she was not having consistent diazepam via a nasogastric tube until the bridle nasogastric tube was inserted 13 days later. We then waited for another 6 days awaiting her response to the diazepam and to allow her to become biochemically stable especially regarding her serum potassium and phosphate. Moreover, we were awaiting completion of all physical tests, particularly those that would rule out intracranial lesions such as the CT scan of the head and bedside nasoendoscopy. In addition, ethical, legal and logistical implications of ECT also contributed to the delay. She was an informal patient and her capacity to consent to ECT was in doubt even with the use of an interpreter. Thus, she was only put on Section 3 after a second opinion was sought. The ECT was administered under Section 62 of the Mental Health Act as an emergency treatment in the general hospital operating theatre rather than the ECT suite. Section 62 had to be used 
while we were awaiting the second opinion approved doctor assessment under Section 58 of the Mental Health Act.

\section{Treatment options for catatonia}

Her relatively poor response to diazepam makes it worthwhile to discuss the available treatments for catatonia. Evidence for various treatment regimens for catatonia has come mainly from case reports and retrospective studies rather than randomised trials.

Benzodiazepines and specifically lorazepam have been found to be effective in $32-80 \%$ of patients. ${ }^{6,7}$ Features of individuals who respond poorly to lorazepam include longterm untreated catatonia, ${ }^{6,8}$ presence of mutism, ${ }^{6}$ a primary diagnosis of schizophrenia (50\% response rate), ${ }^{7}$ presence of third-person auditory hallucinations and passivity phenomena, ${ }^{6}$ and being from a rural background. ${ }^{6}$ Features of individuals who respond well include a primary diagnoses of affective disorders ( $>80$ response rate), ${ }^{7}$ schizoaffective disorders $\left(70 \%\right.$ response rate $\left.^{7}\right)$ and presence of waxy flexibility. ${ }^{7}$ The issue of differential efficacies of various benzodiazepines has not been carefully studied. Nevertheless, a case report of catatonia secondary to paraneoplastic encephalitis was suggestive of lorazepam being more effective than diazepam. ${ }^{9}$ Overall, benzodiazepines are used as a first-line treatment. They are remarkably safe but care should be taken regarding (a) hypoventilation in individuals who are obese or those with obstructive apnoea; and (b) falls in elderly people or in patients that had been in prolonged stupor because of problems with balance. There is also a small risk of previously immobile patients being switched to the excited form of catatonia. ${ }^{10}$

Electroconvulsive therapy has been found to be effective in more than $90 \%$ of patients with acute catatonia irrespective of primary diagnoses..$^{10,11}$ It might be less effective in chronic catatonia and treatment may require many sessions. ${ }^{12}$ In spite of proven effectiveness as a firstline therapy without major adverse effects, ${ }^{13}$ ECT is routinely used as a second-line treatment. This is mainly because of social stigma and excessive concern regarding potential retrograde amnesia. ${ }^{10}$ Nevertheless, ECT is the recommended first-line treatment for malignant catatonia (characterised by severe rigidity, autonomic nervous system instability and altered mental status). ${ }^{14}$ Moreover, the response of rare gastrointestinal features of catatonia, such as dysphagia, to ECT rather than benzodiazepines has been reported. ${ }^{5}$

The use of antipsychotics to treat catatonia is rather controversial. This is because antipsychotics might be a risk factor for precipitation of neuroleptic malignant syndrome; $;^{7,15}$ especially in patients who are catatonic with low serum ferritin. ${ }^{7,16}$ Furthermore, others have considered neuroleptic malignant syndrome as a subtype of malignant catatonia rather than a separate clinical entity (iatrogenic catatonia). ${ }^{17}$ Unfortunately, it is the patients with schizophrenia whose mainstays of treatment are antipsychotics that respond relatively poorly to benzodiazepines. Nevertheless, there are recent case reports of the use of atypical antipsychotics, such as clozapine ${ }^{18}$ and olanzapine, ${ }^{19}$ in the treatment of recurrent and periodic catatonia respectively. These are the subtypes of catatonia that have a diminishing response to benzodiazepines and ECT with each recurrence. ${ }^{18}$
Amantadine (a $\mathrm{N}$-methyl-D-aspartate receptor antagonist) has also been found useful in a similar category of patients. ${ }^{20}$ Overall, it has been recommended that antipsychotics should only be used in those that have failed to respond to sequential treatment with benzodiazepines and ECT. ${ }^{7}$ Other medications that have been used in treating catatonia with anecdotal successes include bromocriptine (a dopamine agonist), dantrolene, calcium channel blockers, carbamazepine, lithium, levothyroxine, amobarbital and corticosteroids. $^{21}$

\section{Implications}

In our case study the arrival at the diagnosis required interdisciplinary collaboration among psychiatrists, gastroenterologists, neurologists, otolaryngologists, speech and language therapists, radiologists, nurses and professional sign language interpreters. Accurate diagnosis of catatonia in this group of individuals is important as 1 in 7 of the UK population (about 9 million people) are either deaf or have a significant hearing problem. ${ }^{22}$ British Sign Language is the preferred language of 50000-70000 people within the $\mathrm{UK}^{23}$ The latter group probably represents the deaf-mute population in the UK. It is estimated that between 1 and $2 \%$ of the population develop bipolar disorder in their lifetime. ${ }^{24}$ At least $50-63 \%$ of patients with bipolar disorder require admission once ${ }^{25}$ and $25 \%$ of the admitted patients develop catatonia. ${ }^{26}$ Thus, approximately 142 cases and 1 case of catatonia per year occur among those with significant hearing problems and those that prefer to use British Sign Language respectively.

\section{About the authors}

Gbolagade Sunmaila Akintomide is an ST4 psychiatric trainee at Central and North West NHS Foundation Trust (formerly Betsi Cadwaladr University Health Board). Stuart Williams Porter and Anita Pierce are consultant general adult psychiatrists at Glan Clwyd Hospital, Betsi Cadwaladr University Health Board, Bodelwyddan, North Wales.

\section{References}

1 Bruni B, Carlini M, Gamba S, Giobbe D, Nobili M. Catatonia with high CSF lactate in a case of diabetes with associated conditions. Acta Diabetol Lat 1980; 17: 273-6.

2 American Psychiatric Association. Diagnostic and Statistical Manual of Mental Disorders (4th edn, text revision) (DSM-IV-TR). APA, 2000.

3 Suzuki H, Fukushima T, Makino K, Kuwabara T. Patient with encephalitis presenting with olanzapine-responsive malignant catatonia [in Japanese]. Rinsho Shinkeigaku 2010; 50: 329-31.

4 Dalò V, Lavatelli L, Zeppegno P, Torre E. Catatonia: a case report. Eur Psychiatry 2010; 25 (suppl 1): 1163.

5 Kaufmann RM, Schreinzer D, Strnad A, Mossaheb N, Kasper S, Frey R. Case report: intestinal atonia as an unusual symptom of malignant catatonia responsive to electroconvulsive therapy. Schizopr Res 2006; 84: $178-9$.

6 Narayanaswamy JC, Tibrewal P, Zutshi A, Srinivasaraju R, Math SB. Clinical predictors of response to treatment in catatonia. Gen Hosp Psychiatry 2012; 34: 312-4.

7 Rosebush PI, Mazurek MF. Catatonia and its treatment. Schizophr Bull 2010; 36: 239-42.

8 Ungvari GS, Chiu HFK, Chow LY, Lau BST, Tang WK. Lorazepam for chronic catatonia: a randomized, double-blind, placebo-controlled cross-over study. Psychopharmacology 1999; 142: 393-8. 
9 Kaestner F, Mostert C, Behnken A, Boeckermann I, Ternes F, Diedrich M, et al. Therapeutic strategies for catatonia in paraneoplastic encephalitis. World J Biol Psychiatry 2008; 9: 236-40.

10 Bhati MT, Datto CJ, O'Reardon JP. Clinical manifestations, diagnosis, and empirical treatments for catatonia. Psychiatry (Edgmont) 2007; 4: 46-52.

11 Rohland BM, Carroll BT, Jacoby RG. ECT in the treatment of the catatonic syndrome. J Affect Disord 1993; 29: 255-61.

12 Malur C, Pasol E, Francis A. ECT for prolonged catatonia. J ECT 2001; 17 55-9.

13 Maletzky BM. The first-line use of electroconvulsive therapy in major affective disorders. J ECT 2004; 20: 112-7.

14 Fink M. Catatonia and ECT: Meduna's biological antagonism hypothesis reconsidered. World J Biol Psychiatry 2002; 3: 105-8.

15 White $\mathrm{DA}$, Robins $\mathrm{AH}$. Catatonia: harbinger of the neuroleptic malignant syndrome. Br J Psychiatry 1991; 158: 419-21.

16 Lee JW. Serum iron in catatonia and neuroleptic malignant syndrome. Biol Psychiatry 1998; 44: 499-507.

17 Fink M, Taylor MA. Neuroleptic malignant syndrome is malignant catatonia, warranting treatments efficacious for catatonia. Prog Neuropsychopharmacol Biol Psychiatry 2006; 30: 1182-3.

18 Hung YY, Yang PS, Huang TL. Clozapine in schizophrenia patients with recurrent catatonia: report of two cases. Psychiatry Clin Neurosci 2006; 60: $256-8$.
19 Guzman CS, Myung VH, Wang YP. Treatment of periodic catatonia with olanzapine: a case report. Rev Bras Psiquiatr 2007; 29: 380.

20 Hervey WM, Stewart JT, Catalano G. Treatment of catatonia with amantadine. Clin Neuropharmacol 2012; 35: 86-7.

21 Rosebush PI, Hildebrand AM, Furlong BG, Mazurek MF. Catatonic syndrome in a general psychiatric inpatient population: frequency, clinical presentation, and response to lorazepam. J Clin Psychiatry 1990; 51: 357-62.

22 Deafness Research UK. Deafness - The Facts. Deafness Research UK, 2009 (http://www.deafnessresearch.org.uk/factsheets/deafness-thefacts. pdf).

23 British-sign.co.uk. What is British Sign Language? British-sign.co.uk, 2012 (http://www.british-sign.co.uk/what_is_british_sign_language.php).

24 ten Have M, Vollebergh W, Bijl R, Nolen WA. Bipolar disorder in the general population in The Netherlands (prevalence, consequences and care utilisation): results from The Netherlands Mental Health Survey and Incidence Study (NEMESIS). J Affect Disord 2002; 68: 203-13.

25 De Zelicourt M, Dardennes R, Verdoux H, Gandhi G, Khoshnood B Chomette $\mathrm{E}$, et al. Frequency of hospitalisations and inpatient care costs of manic episodes: in patients with bipolar I disorder in France. Pharmacoeconomics 2003; 21: 1080-90.

26 Taylor MA, Abrams R. Catatonia: prevalence and importance in the manic phase of manic-depressive illness. Arch Gen Psychiatry 1977; 34 1223-5.

\title{
Enhancing the effectiveness of community drug and alcohol teams working with opioid-dependent pregnant women: results of an audit
}

\author{
Mani Sairam, ${ }^{1}$ Rakesh Magon, ${ }^{2}$ Christos Kouimtsidis ${ }^{3}$
}

The Psychiatrist (2012), 36, 421-423, doi: 10.1192/pb.bp.111.038166

${ }^{1}$ Beech Low Security Forensic Rehabilitation Unit, Kingsley Green Hospital; ${ }^{2}$ Oxford House Community Mental Health Team, Bishops Stortford; ${ }^{3}$ Hertfordshire Partnership NHS Foundation Trust, St Albans

\section{Correspondence to Christos}

Kouimtsidis

(drckouimtsidis@hotmail.com)

First received 5 Dec 2011, final revision 3 Feb 2012, accepted 9 May 2012
Aims and method To evaluate the quality of services offered by community drug and alcohol teams (CDATs) to pregnant women in substitution treatment. A full audit of the practice across all local CDATs against national standards was undertaken in 2008 and 2010.

Results Quality of services improved and met three standards in 100\% and the fourth standard in $96 \%$ of cases. There was good implementation of the recommendations arising out of the action plan of the first cycle, which resulted in significant improvements in interagency liaison and risk-benefit analysis documentation within the CDATs.

Clinical implications Management of pregnant women in substitution treatment can be improved by adhering to a multipronged approach as identified by this audit.

Declaration of interest All authors were members of the Addiction Services in Hertfordshire when this audit took place.
The extent of problem drinking and drug use in pregnancy is difficult to estimate accurately owing to associated stigma and secrecy, and it is probably underestimated. A quarter of those presenting to drug misuse agencies are women of childbearing age. ${ }^{1}$ A national household survey in the USA on drug use and health (2007-2008) showed $5.1 \%$ of pregnant women using illicit drugs in the past month (including cocaine). ${ }^{2}$ The recent confidential enquiry into maternal deaths highlighted drug- and/or alcohol-related deaths in this population. ${ }^{3}$ Substance misuse was directly or indirectly related to death in 57 out of 295 reported deaths (12 alcohol dependent; 45 illicit drugs dependent). The 